\title{
Purification of Synaptic Ribbons, Structural Components of the Photoreceptor Active Zone Complex
}

\author{
Frank Schmitz, Martin Bechmann, and Detlev Drenckhahn \\ Institute of Anatomy, University of Würzburg, D-97070 Würzburg, Germany
}

Synaptic ribbons are plasma membrane-associated structural elements in photoreceptor synaptic terminals. They seem to act as high capacity "docking sites" of synaptic vesicles that provide the fusion sites of the photoreceptor synapse ("active zones"), with a large supply of immobilized synaptic vesicles rapidly available for exocytosis. Synaptic ribbons are regarded as a specialized type of presynaptic densities found in virtually all synapses. The molecular composition of presynaptic densities and synaptic ribbons is unknown. The aim of this study was the isolation of synaptic ribbons from photoreceptor synapses. For this purpose, we first isolated a membrane fraction from the bovine retina that was strongly enriched in photoreceptor synapses. From this fraction, a Triton X-100-resistant subfraction was purified that consisted mainly of synaptic ribbons and their disassembly products. The high enrichment of synaptic ribbons was verified by electron microscopy and immunolabeling using an antibody that specifically binds to synaptic ribbons. SDSPAGE analysis of this synaptic ribbon fraction displayed several major polypeptide bands migrating at $\sim 240,60,55,43$, and 30 $\mathrm{kDa}$. The purification procedure described here is a first promising step toward the identification of the yet unknown constituents of synaptic ribbons from photoreceptor synapses and possibly also of presynaptic densities from other synapses.

Key words: synaptic ribbons; presynaptic densities; retina; photoreceptor synapse; active zones; exocytosis
The vertebrate retina contains two circumscribed layers in which synaptic transmission takes place: the outer plexiform layer (OPL) and the inner plexiform layer (IPL). Synaptic transmission in the OPL of the retina occurs mainly between presynaptic terminals of rod and cone photoreceptors and the postsynaptic dendrites of bipolar and horizontal cells. Most typically, a single large presynaptic terminal of a photoreceptor cell contacts a great number of postsynaptic dendrites (for review, see Dowling, 1987). These many postsynaptic dendrites are accommodated within an invagination of the presynaptic photoreceptor terminal. By this means, a synaptic cavity is created in which the postsynaptic dendrites form a plexus. A typical landmark of the active zones of photoreceptor presynaptic terminals is conspicuous presynaptic densities, the synaptic ribbons (Sjöstrand, 1958). In conventional electron micrographs, synaptic ribbons appear as bar-shaped structures, with one end anchored at the plasma membrane and the other end pointing into the cytoplasm (for review, see Dowling, 1987). Reconstructions of serial ultrathin sections demonstrated that this bar-like profile of synaptic ribbons resulted from cross-sections of a structure that was actually plate-like (Sjöstrand, 1958, McCartney and Dickson, 1985; Rao-Mirotznik et al., 1995). Furthermore, this plate-like structure was shown to be bent around the synaptic cavity, thus giving rise to a horseshoe-shaped appearance of the synaptic ribbons in three-dimensional representations (Sjöstrand, 1958; McCartney and Dickson, 1985; RaoMirotznik et al., 1995). Along its entire surface, the synaptic ribbon is associated with synaptic vesicles that are attached to the

\footnotetext{
Received Feb. 5, 1996; revised Aug. 26, 1996; accepted Sept. 3, 1996.

This study was supported by research grants from the Deutsche Forschungsgemeinschaft and the Fonds der Chemischen Industrie to F.S. and D.D. We thank Tanja Geißendörfer and Christine Hambrecht for excellent technical assistance.

Correspondence should be addressed to Dr. Frank Schmitz at his present address: Max-Planck-Institut für experimentelle Medizin, Abteilung: Molekulare Neurobiologie, Hermann-Rein-Straße 3, 37075 Göttingen, Germany.

Copyright (C) 1996 Society for Neuroscience $0270-6474 / 96 / 167109-08 \$ 05.00 / 0$
}

ribbons via stalk-like connections (McLaughlin and Boykins, 1977). Exocytosis of synaptic vesicles in the photoreceptor synapse occurs at plasma membrane patches in close contact with the membrane-anchored end of the ribbon (Raviola, 1976; Schaeffer and Raviola, 1978; Schaeffer et al., 1982; for review, see Burns and Augustine, 1995). Therefore, the ribbon has been assumed to be important for vesicle recruitment at the active zone of photoreceptor synapses (for review, see Burns and Augustine, 1995; Rao-Mirotznik et al., 1995). Cone photoreceptor synapses contain several active zones and thus several synaptic ribbons (Ahnelt et al., 1990), whereas rod photoreceptor synapses usually contain only a single synaptic ribbon and a single active zone (RaoMirotznik et al., 1995). Synaptic ribbons also occur in synapses of the IPL of the retina, although the ribbons are much smaller and less abundant at this location (for review, see Dowling, 1987). Synaptic ribbons in photoreceptors of various species are known to be dynamic structures that are able to disassemble and reassemble (Wagner, 1973; Schmitz et al., 1989; Schmitz and Drenckhahn, 1993). The molecular composition of synaptic ribbons is unknown.

In a preliminary report (Schmitz et al., 1995), we described a rabbit autoantibody that binds to synaptic ribbons of photoreceptors in several mammalian species. In the present study, we used this antibody to develop a protocol for the purification of synaptic ribbons from isolated photoreceptor synapses of the bovine retina as a first step toward the molecular characterization of this important structure of the photoreceptor active zone complex.

\section{MATERIALS AND METHODS}

Retinae. Bovine eyes were obtained from a local slaughterhouse. Eyes were transported to the laboratory on ice, and the retinae were isolated and processed within $30 \mathrm{~min}$ postmortem. Retinae from adult Wistar rats were prepared within $10 \mathrm{~min}$ postmortem. A human retina was obtained from the Department of Ophthalmology (University of Würzburg) approximately $60 \mathrm{~min}$ after an eye enucleation surgery of a patient who had an ocular melanoma. 
Antibodies and antisera. A mouse monoclonal antibody directed against synaptophysin, a kind gift from Dr. B. Wiedenmann (Wiedenmann and Franke, 1985), was used at a 1:100 dilution in PBS, pH 7.4. A mouse monoclonal antibody against glial fibrillary acidic protein (GFAP) (clone G-A-5) was purchased from Sigma (Deisenhofen, Germany) and used at a dilution in PBS of 1:100 for immunofluorescence and 1:10,000 for immunoblotting. A monoclonal antibody against SP14 (Honer et al., 1993; Simpson et al., 1994), which is probably a SNAP25-like molecule (for a review, see Rothman, 1994), was obtained from Biermann (Bad Nauheim, Germany) and used for immunoblotting at a dilution of 1:200 in PBS. The antibody used in this study for labeling photoreceptor synaptic ribbons (see below) was present in the serum of a rabbit that had been immunized against the rat erythrocyte anion exchanger (AE1, "band 3") (Drenckhahn et al., 1985). The component of synaptic ribbons that was detected by this antiserum in the retina was not related to AE1, but was attributable to an autoantibody directed against a yet unknown retinal molecule for the following reasons. (1) Affinity purification of the serum with AE1 cut out from nitrocellulose sheets after electrophoretic transfer (Olmstedt, 1981) abolished staining of the OPL but not staining of erythrocytes; (2) preabsorption of the serum with these AE1containing nitrocellulose sheets eliminated labeling of erythrocytes but not of synapses; and (3) other polyclonal rabbit antisera directed against AE1 of rat, pig, and human erythrocytes did not stain photoreceptor synapses, although they brightly stained erythrocytes. For simplicity, this serum that labeled synaptic ribbons is called "ribbon antiserum" in the following text. Key experiments were carried out with both unfractionated serum and serum preabsorbed with rat AE1 (see above). The success of preabsorption was checked by immunolabeling of rat blood smears with preabsorbed and nonabsorbed ribbon antiserum. The ribbon antiserum was used at a 1:50 dilution in PBS, pH 7.4.

Immunofluorescence microscopy. Retinae were processed for immunocytochemistry by flash-freezing in liquid nitrogen-cooled isopentane. Cryostat sections (5 $\mu \mathrm{m}$ in thickness) were cut with a Frigocut $2800 \mathrm{E}$ (Reichert-Jung, Nußloch, Germany). Cryosections were thawed on polyL-lysine-coated coverslides and dried on a heating plate $\left(37^{\circ} \mathrm{C}\right)$ for $1 \mathrm{hr}$. Incubation with primary antibodies (diluted as indicated above) was performed overnight at $4^{\circ} \mathrm{C}$. After several washes with $\mathrm{PBS}$ to remove unbound antibody, the binding of the primary antibody was visualized with goat anti-rabbit or goat anti-mouse secondary antibodies conjugated to either tetramethyl rhodamine isothiocyanate or fluorescein isothiocyanate (Biotrend, Cologne, Germany) at a 1:100 dilution in PBS, pH 7.4 (1 $\left.\mathrm{hr}, 22^{\circ} \mathrm{C}\right) .1 .5 \% n$-propylgallate $(\mathrm{w} / \mathrm{v})$ was added to the mounting medium (60\% glycerol in PBS) to retard photobleaching of the fluorochromes (Giloh and Sedat, 1982). Sections were analyzed with an Olympus BH2 microscope (Olympus, Hamburg, Germany) equipped with an epifluorescence optical system or with an MRC 600 confocal laser microscope equipped with the respective optical filter systems (Bio-Rad, Munich, Germany). Controls were performed by omitting primary antibodies and by the experiments described above. Immunostained sections observed with conventional epifluorescence microscopy were documented with TMAX 400 film (Kodak, Rochester, NY). The confocal microscopy images were printed with a black and white paper printer (UP-930, Sony, Cologne, Germany)

Transmission electron microscopy of retinal tissue fractions. Aliquots of the different retinal tissue fractions were saved from each step of the fractionation procedure and fixed by adding glutaraldehyde to a final concentration of $5 \%(\mathrm{v} / \mathrm{v})$. Fixation was performed for $3 \mathrm{hr}$ at $4^{\circ} \mathrm{C}$ with gentle agitation of the samples. After fixation, samples were sedimented by a $10,000 \times g$ spin in an Eppendorff centrifuge for $10 \mathrm{~min}$. After several washes with $\mathrm{PBS}$, the pellets were post-fixed with $1 \% \mathrm{OsO}_{4}$ in $\mathrm{H}_{2} \mathrm{O}(\mathrm{w} / \mathrm{v})$ for $1 \mathrm{hr}$ at $4^{\circ} \mathrm{C}$. The samples were block-contrasted with $2 \%$ uranyl acetate in $\mathrm{H}_{2} \mathrm{O}(\mathrm{w} / \mathrm{v})$ for $3 \mathrm{hr}$ at $4^{\circ} \mathrm{C}$, dehydrated with an ascending ethanol concentration series, and embedded in a resin mixture containing $49.6 \%$ glycid ether (1,2,3-propanetriol glycidyl ether, epoxy equivalent of $150)(\mathrm{w} / \mathrm{w}), 21 \%$ 2-dodecenylsuccinic acid anhydride (w/w), 29\% (w/w) methylnadic anhydride, and $0.4 \%$ (w/w) 2,4,6-tris(dimethylaminomethyl)phenol (Serva, Heidelberg, Germany). The resin was polymerized at $60^{\circ} \mathrm{C}$ for $12 \mathrm{hr}$. Sections were cut with a Reichert Ultracut E (ReichertJung). Sections were analyzed with a Zeiss EM109 and photographed with Agfa Ortho 25 film (Agfa-Gevaert, Leverkusen, Germany).

Immunoelectron microscopy. A preembedding protocol was used for the ultrastructural localization of the antigen detected by the ribbon antiserum. For immunolabeling, both cryostat sections from whole retina and cryostat sections from retinal fractions prepared as described above were used. Twenty-micrometer-thick cryostat sections were thawed on poly-L- lysine-coated coverslides. These sections either were processed directly for immunostaining or first gently fixed with $0.1 \%$ formaldehyde in PBS for $10 \mathrm{~min}\left(22^{\circ} \mathrm{C}\right)$. Before incubation with the primary antibody, sections were preincubated with PBS containing $1 \%(\mathrm{w} / \mathrm{v})$ bovine serum albumin (BSA) for $1 \mathrm{hr}$ at $22^{\circ} \mathrm{C}$ to block nonspecific protein binding sites. After this blocking step, sections were incubated with ribbon antiserum at a dilution of 1:50 in PBS (overnight at $4^{\circ} \mathrm{C}$ ). Unbound antibody was removed by several washes with PBS $(5 \times 20 \mathrm{~min}$ at room temperature $)$. Binding of the primary antibody was visualized by either goat anti-rabbit secondary antibodies (Amersham, Braunschweig, Germany) coupled to 5 $\mathrm{nm}$ gold particles (1:40 dilution in PBS, incubation for overnight at $4^{\circ} \mathrm{C}$ ) or by goat anti-rabbit secondary antibodies (Biotrend, Cologne, Germany) coupled to ultra-small $(0.8 \mathrm{~nm})$ gold particles (1:40 dilution in PBS, overnight at $\left.4^{\circ} \mathrm{C}\right)$. After several washes with PBS $(5 \times 20 \mathrm{~min}$ at $22^{\circ} \mathrm{C}$ ), samples were fixed with a $5 \%$ glutaraldehyde solution in PBS (1 hr at $\left.22^{\circ} \mathrm{C}\right)$. The slides were post-fixed with $1 \% \mathrm{OsO}_{4}$ in $\mathrm{H}_{2} \mathrm{O}(\mathrm{w} / \mathrm{v})$ for $2 \mathrm{hr}$ (at $4^{\circ} \mathrm{C}$ ) and contrasted with $2 \%$ uranyl acetate in $\mathrm{H}_{2} \mathrm{O}(\mathrm{w} / \mathrm{v})$ for $2 \mathrm{hr}$ (at $4^{\circ} \mathrm{C}$ ). If the secondary antibodies conjugated to ultra-small gold particles were used, a silver-enhancement step was performed after osmification of the samples, exactly as described by Danscher (1981) for $30 \mathrm{~min}$ at $22^{\circ} \mathrm{C}$ in the dark. After dehydration in an ascending ethanol concentration series and transfer through propylene oxide, samples were embedded in the resin mixture described above. From these, ultrathin sections were cut with a Reichert Ultracut E (Reichert-Jung) and analyzed with the Zeiss EM 109. As controls, cryostat sections were treated and incubated exactly as described above but without the addition of ribbon antiserum.

SDS-PAGE, immunoblotting, and determination of protein concentrations. SDS-PAGE was performed on $10 \%$ polyacrylamide gels as described by Laemmli (1970). Immunoblotting was carried out according to Towbin et al. (1979). Proteins electrotransferred to nitrocellulose were probed with the antibodies diluted in PBS, as described above. After specific protein binding sites were blocked with $5 \%$ skim milk in PBS for $1 \mathrm{hr}$ at room temperature, binding of primary antibodies was detected by a $1 \mathrm{hr}$ incubation with goat anti-mouse $\mathrm{IgG} / \mathrm{IgM}$ secondary antibodies conjugated to peroxidase and diluted 1:200 in PBS (Jackson Immunoresearch Laboratories, West Grove, PA). As chromogen 4-chloronaphthol (BioRad, Munich, Germany) was used according to the manufacturer's instructions. The following molecular weight markers were used: (1) membrane proteins of erythrocyte ghosts, (2) molecular weight markers from Boehringer Mannheim (Combithek; Mannheim, Germany), and (3) molecular weight markers from Sigma ("wide range", M4038).

Protein concentration was determined by a modification of the method of Heinzel et al. (1965) (Dieckmann-Schuppert and Schnittler, in press). Ten microliters of membrane protein samples dissolved in Laemmli sample buffer were applied onto cellulose acetate strips (Schleicher and Schüll, Darmstadt, Germany) of uniform areas $\left(1.5 \mathrm{~cm}^{2}\right)$. The strips were dried and stained in solution containing $0.5 \%$ amidoblack, $45 \%$ methanol, and $10 \%$ glacial acetic acid in $\mathrm{H}_{2} \mathrm{O}\left(10 \mathrm{~min}, 22^{\circ} \mathrm{C}\right)$. Unbound dye was removed by several washes with $47.5 \%$ methanol $(\mathrm{v} / \mathrm{v})$ and $5 \%$ glacial acetic acid (v/v) in $\mathrm{H}_{2} \mathrm{O}$. The stained cellulose acetate strips were dissolved in $1 \mathrm{ml}$ of $80 \%$ formic acid (v/v), $10 \%$ glacial acetic acid (v/v), and $10 \% \mathrm{TCA}\left(\mathrm{w} / \mathrm{v}\right.$, final concentration) in $\mathrm{H}_{2} \mathrm{O}$ for $30 \mathrm{~min}$ at $50^{\circ} \mathrm{C}$. Samples were measured at $620 \mathrm{~nm}$ against blank Laemmli buffer without added proteins. Defined concentrations of BSA $(40,20,10,5,2.5$, and $1.25 \mu \mathrm{g}$ of protein in $10 \mu \mathrm{l}$ of Laemmli sample buffer, respectively) were used as standards. Correlation coefficients of the regression curve obtained with these standards was usually better than 0.995 .

Purification of photoreceptor synaptic complexes ("OPL-fraction”). As a first step in the purification of photoreceptor synapses, a crude synaptic membrane fraction was prepared as described previously (Schmitz et al., 1993). Briefly, retinae freshly isolated from bovine eyes (obtained from a local slaughterhouse within $30 \mathrm{~min}$ postmortem) and detached from pigment epithelium were disrupted by shear forces exerted by an Ultraturrax for 3 min on ice (Type TP 18/10; Janke and Kunkel, Staufen, Germany) in hypotonic homogenization buffer containing $15 \mathrm{~mm}$ $\mathrm{Na}_{2} \mathrm{HPO}_{4}$, pH 7.4, $1 \mathrm{~mm}$ EGTA, $1 \mathrm{~mm} \mathrm{MgCl}_{2}$, and $1 \mathrm{~mm}$ phenylmethylsulfonyl fluoride for $3 \mathrm{~min}$ at $4^{\circ} \mathrm{C}$. Thirty-five milliliters of homogenization buffer were used for eight isolated retinae. For preparation of crude synaptic membranes, $20 \mathrm{ml}$ of homogenate was overlayered on $10 \mathrm{ml}$ of a sucrose cushion containing $50 \%$ sucrose $(w / v)$ in homogenization buffer and centrifuged for $50 \mathrm{~min}$ at $15,000 \mathrm{rpm}\left(\sim 27,200 \times g_{\max }\right)$ at $4^{\circ} \mathrm{C}$ in a JA20 rotor (Beckman, Palo Alto, CA). At the interface between the sucrose cushion and the supernatant, a broad opaque band of membranes was visible and used for the subsequent purification of photoreceptor synapses. This band was removed carefully with a Pasteur pipette and 
diluted with approximately twofold its volume with homogenization buffer. This diluted suspension was spun in a JA20 rotor at 20,000 rpm $\left(\sim 48,400 \times g_{\max }\right)$ for $10 \mathrm{~min}\left(4^{\circ} \mathrm{C}\right)$. The supernatant was discarded, and the pellet was resuspended with approximately the same volume of homogenization buffer. For convenience, this resulting membrane suspension containing crude synaptic membranes (CSMs) was denoted CSM-fraction. The CSM-fraction was overlayered on a linear sucrose gradient ranging from 35 to $50 \%$ sucrose $(\mathrm{w} / \mathrm{v})$ in homogenization buffer. Membranes were spun at $13,000 \mathrm{rpm}\left(\sim 30,000 \times g_{\max }\right)$ for $1.5 \mathrm{hr}$ at $4^{\circ} \mathrm{C}$ in an SW40 rotor. After this spin, two bands and a large pellet were visible. A membrane fraction that was recovered as a broad band at a sucrose density of $\sim 40 \%(\mathrm{w} / \mathrm{v})$ in homogenization buffer (sucrose density calculated by its distance between the top and bottom of the gradient) was denoted OPL-fraction and characterized as described below. Membrane fractions were analyzed by immunoblotting and immunofluorescence microscopy with the ribbon antiserum. To analyze the retinal fractions by immunofluorescence, microscopy samples were diluted with the twofold volume of homogenization buffer, sedimented in an Eppendorf centrifuge (model 5415C, Eppendorf, Hamburg, Germany) at 14,000 $\mathrm{rpm}\left(\sim 15,900 \times g_{\max }\right)$ at $4^{\circ} \mathrm{C}$ for $10 \mathrm{~min}$, and flash-frozen in liquid nitrogen. From these frozen samples, $10-\mu$ m-thick cryostat sections were cut and immunolabeled with the ribbon antiserum as described above.

Purification of synaptic ribbons from the isolated photoreceptor synapses of the OPL-fraction. OPL membranes were diluted with the twofold volume of homogenization buffer and spun in a JA20 rotor at $11,000 \mathrm{rpm}$ $\left(\sim 14,600 \times g_{\max }\right)$ for $10 \mathrm{~min}$ at $4^{\circ} \mathrm{C}$. The resulting sediment was resuspended in homogenization buffer containing $1 \%$ Triton X-100 (w/v) to a protein concentration of $\sim 1 \mathrm{mg} / \mathrm{ml}$. The pellet was homogenized three times with a tight-fitting Teflon pestle and kept on ice for $\sim 30 \mathrm{~min}$. After this incubation period, the Triton-insoluble fraction of photoreceptor synapses was sedimented in a JA20 rotor at 11,000 $\mathrm{rpm}\left(\sim 14,600 \times g_{\max }\right)$ for $10 \mathrm{~min}$ at $4^{\circ} \mathrm{C}$. The sediment was resuspended with approximately the same volume of homogenization buffer containing $20 \%$ sucrose. This suspension was overlayered on a sucrose step gradient containing $2 \mathrm{ml}$ of each of the following sucrose concentrations (in homogenization buffer): $30,40,50$, and $70 \%$. Then the sample was centrifuged in an SW40 rotor at $11,000 \mathrm{rpm}\left(\sim 20,000 \times g_{\max }\right)$ for $75 \mathrm{~min}$ at $4^{\circ} \mathrm{C}$. The opaque protein bands at the respective interfaces of the sucrose step gradient were tested for the presence of synaptic ribbons by immunofluorescence and electron microscopy. The retinal subfraction between the 50 and $70 \%$ sucrose step contained the highest density and purity of synaptic ribbons and was denoted SR-fraction. The entire purification procedure is summarized in Figure 1.

\section{RESULTS}

\section{An autoantibody directed against photoreceptor synaptic ribbons}

The starting point of this study was a rabbit autoantibody that reacted with photoreceptor synaptic ribbons in all mammalian retinae studied, i.e., bovine (Figs. 2, 3), human, and rat (not shown). At the electron microscope level (Fig. 2), the ribbon antiserum was revealed to bind to the surface of synaptic ribbons that were densely labeled with immunogold particles. Ribbons of both rod and cone synapses were labeled. At the light microscope level of cryostat sections of the corresponding retinae processed for immunofluorescence microscopy, most synaptic ribbons in the OPL appeared as horseshoe-shaped structures of $\sim 1-3 \mu \mathrm{m}$ in length (Fig. 3). Groups of synaptic ribbons often located in the vitread half of the OPL (Fig. 3) were tentatively interpreted as belonging to the large cone terminals (pedicles), whereas individual nonclustered synaptic ribbons (Fig. 3) possibly belong to small rod terminals (spherules). All other neuronal structures of the entire retina, except the much smaller synaptic ribbons of synapses from the IPL (Fig. 3b), were not labeled by the ribbon antiserum.

\section{Purification of a retinal photoreceptor synapse fraction}

Bovine retinae detached from pigment epithelium were fractionated as described above. Fractions were pelleted, sectioned, and screened with the ribbon antiserum. Figure $4 a$ represents the

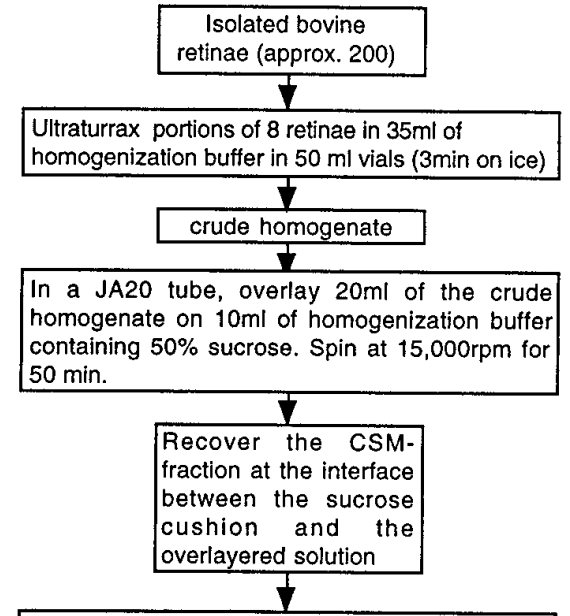

Dilute the CSM-fraction with approx. the 2fold volume of homogenization buffer. Spin at $20,000 \mathrm{rpm}$ in a JA20 rotor for $10 \mathrm{~min}$. Resuspend the pellet in approx. the same volume of homogenization buffer. Overlay this suspension onto a linear sucrose gradient ranging from $35 \%$ to $50 \%$ sucrose in homogenization buffer. Centrifuge in a SW40 rotor at $13,000 \mathrm{rpm}$ for $75 \mathrm{~min}$.

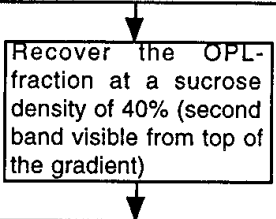

Dilute the OPL-membranes with approx. the 2fold volume of homogenization buffer. Spin in a JA20 rotor at $20,000 \mathrm{rpm}$ for $10 \mathrm{~min}$. Resuspend the resulting pellet in approx. the same volume of homogenization buffer containing $1 \%$ Triton $X$ 100. Incubate on ice for $30 \mathrm{~min}$. Sediment the Triton-insoluble components in a JA20 rotor at $11,000 \mathrm{rpm}$ for $10 \mathrm{~min}$. Resuspend the pellet in approx. the same volume of homogenization buffer containing $20 \%$ sucrose. In a SW40 tube, overlay $2 \mathrm{ml}$ of this solution on a discontinuous sucrose gradient with $2 \mathrm{ml}$ steps of $30 \%, 40 \%$, $50 \%$ and $70 \%$ sucrose in homogenization buffer. resp.. Spin in a SW40 rotor at 11,000 rpm for $75 \mathrm{~min}$ (slow acceleration, no brake).

Recover the SR-fraction
at the interface
between the $50 \%$ - and
$70 \%$ - sucrose cushion.
Yield: approx. $20 \mathrm{mg}$

All steps were performed at $4^{\circ} \mathrm{C}$ if not denoted otherwise.

Figure 1. Flow diagram of the purification procedure of synaptic ribbons from bovine photoreceptor synapses.

starting material, a homogenate of the bovine retina; $4 b$ is an intermediate step of purification, the CSM-fraction; and $4 c$ is the most purified membrane fraction with the highest density of large horseshoe-shaped synaptic ribbons, the OPL-fraction. Very obvious is the strong increase in the number of synaptic ribbons in the OPL-fraction (Fig. 4c) as compared with the starting homogenate (Fig. 4a) and the CSM-fraction (Fig. 4b). The immunostained horseshoe-shaped synaptic ribbons in the OPL-fraction displayed a size and morphology similar to those observed in tissue sections of the retina (Fig. 3). 


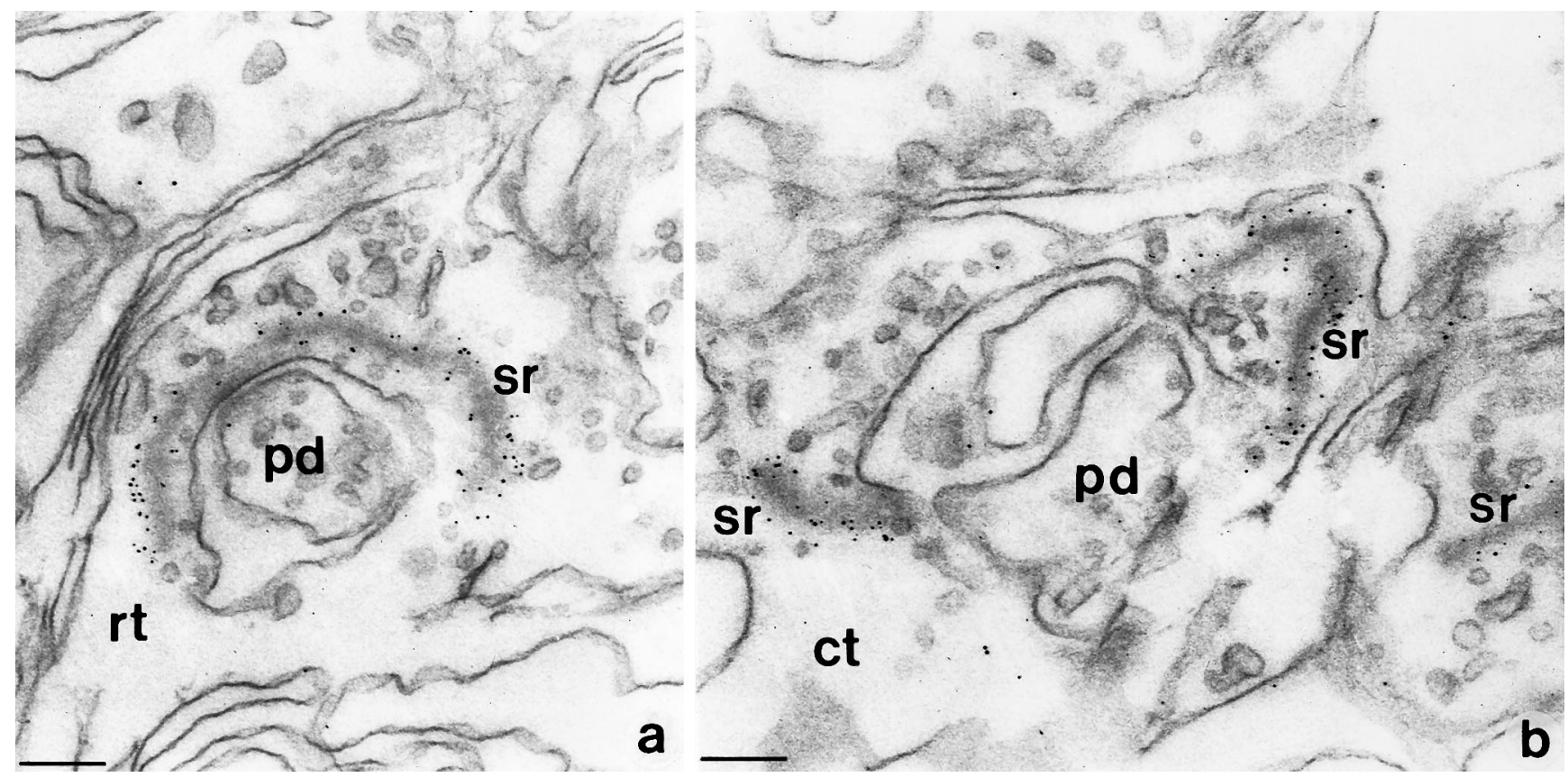

Figure 2. $a, b$, Ultrastructural localization of the binding sites of the ribbon antiserum in the bovine retina. Ribbons in both rod $(a)$ and cone $(b)$ photoreceptor cells are densely labeled by the $5 \mathrm{~nm}$ immunogold complexes, whereas all other structures within the presynaptic terminals are unlabeled. $s r$, Synaptic ribbon; $p d$, postsynaptic dendrites in the photoreceptor synaptic cavity; $r t$, rod presynaptic terminal; $c t$, cone presynaptic terminal (identified by size of synaptic terminal and number of synaptic ribbons). Scale bars, $100 \mathrm{~nm}$.
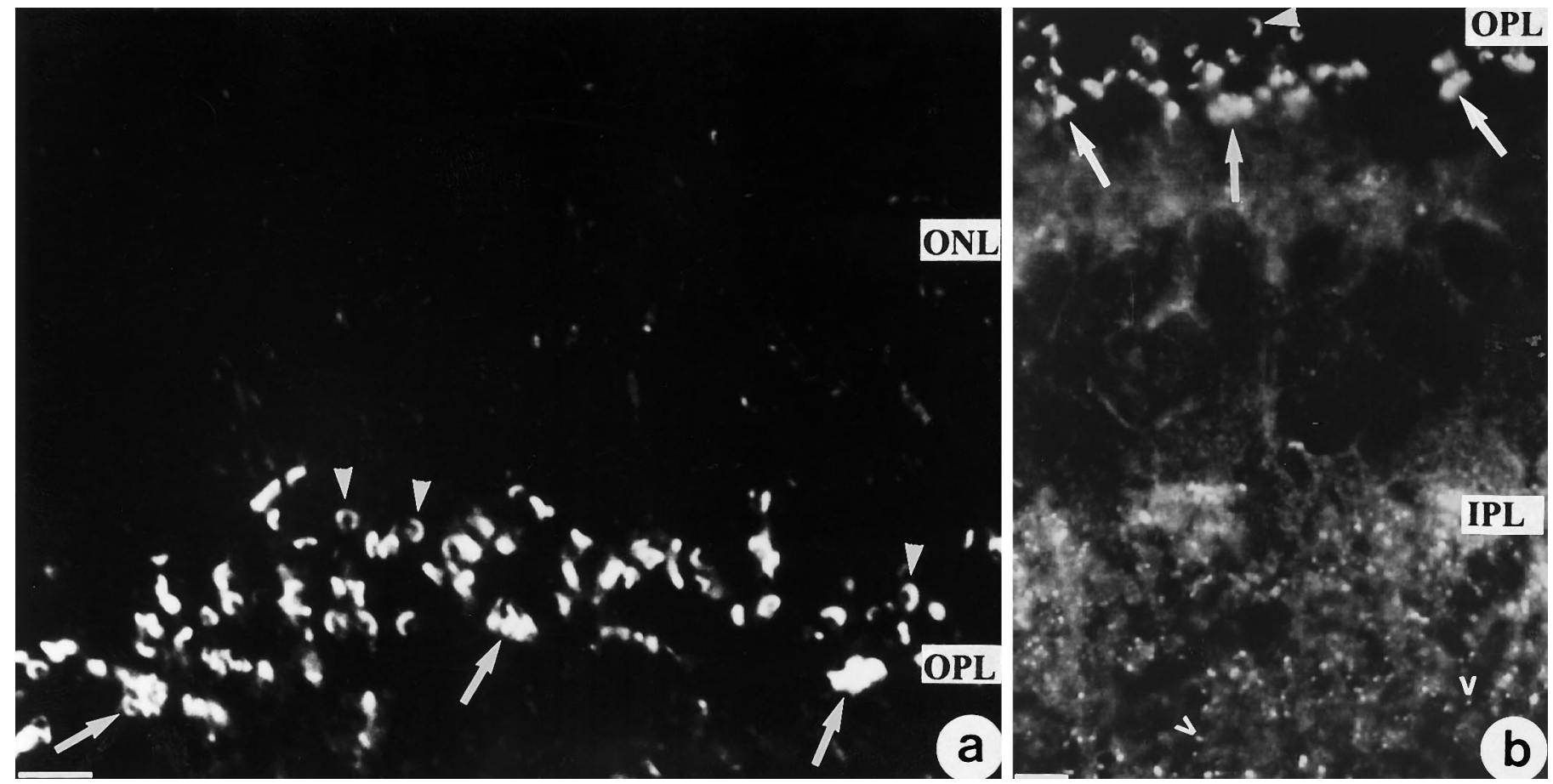

Figure 3. $a, b$, Cryostat section of the bovine retina immunolabeled with the ribbon antiserum. Note large horseshoe-shaped structures in the outer plexiform layer $(O P L)$. Arrows indicate groups of synaptic ribbons typical for cone synapses, and arrowheads denote individual synaptic ribbons typical for rod synapses. Open arrowheads point to small ribbons in synapses of the IPL. ONL, Outer nuclear layer; INL, inner nuclear layer; IPL, inner plexiform layer. Scale bars, $8 \mu \mathrm{m}$.

Electron microscopy of the OPL-fraction also demonstrated the presence of isolated photoreceptor synapses (Fig. 5c). The typical morphology of photoreceptor synaptic ribbons, including synaptic vesicles attached to their surface, was retained. The synaptic cavity was either empty (loss of postsynaptic elements) or still contained postsynaptic dendritic endings. In the latter case, pre- and postsynaptic densities were still preserved. The cytoplasmic space of the synaptosomes seemed to be lighter than in situ, most probably because of the loss of cytosol attributable to the hypotonic homogenization buffer used during the purification proce- 

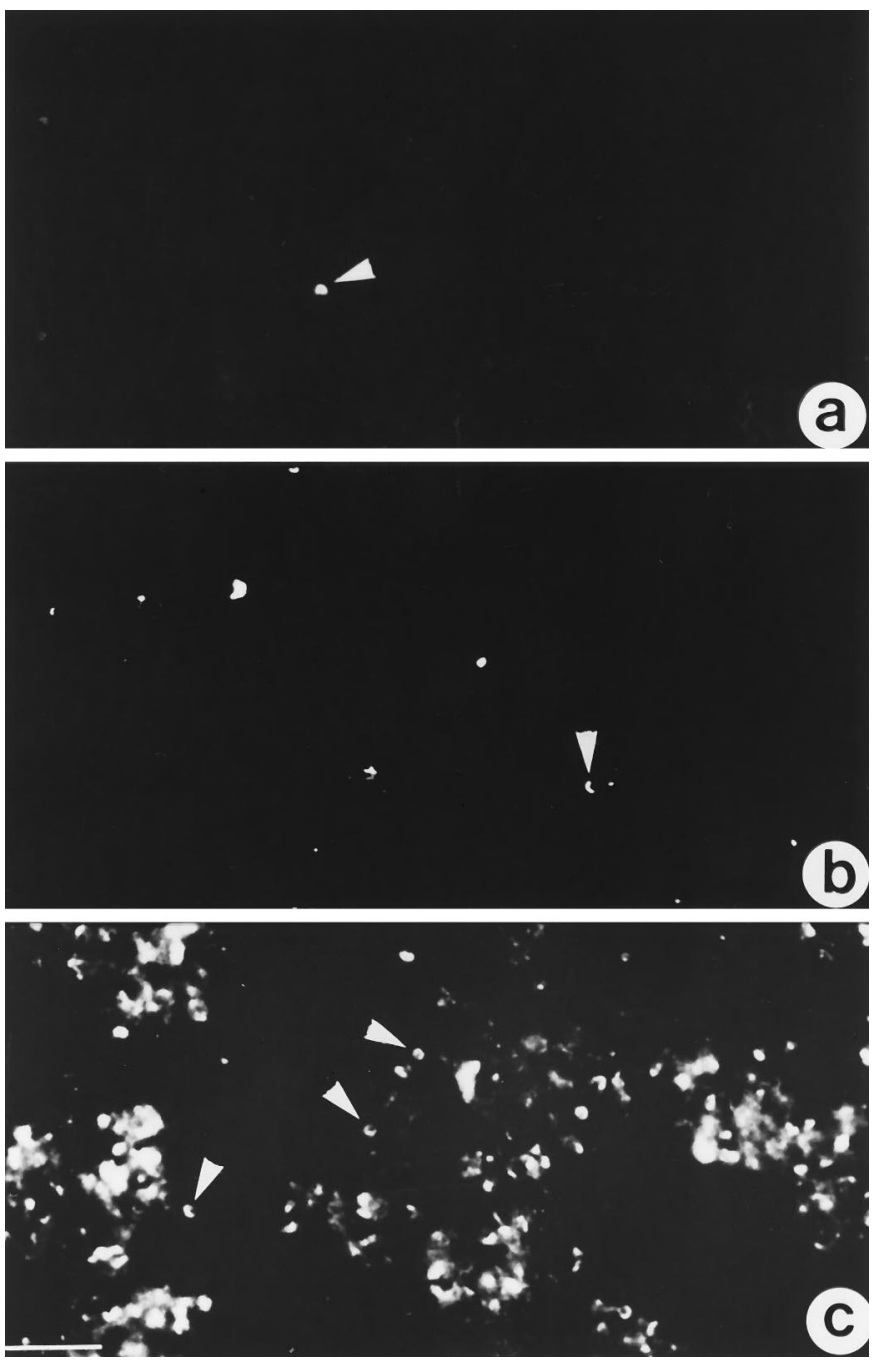

Figure 4. $a-c$, Sections of different retinal fractions immunolabeled with the ribbon antiserum. $a$ represents a confocal micrograph of the starting material (the crude retinal homogenate), $b$ the CSM-fraction, and $c$ the OPL-fraction. Note that synaptic ribbons (arrowheads) are strongly enriched in the OPL-fraction. Scale bar, $15 \mu \mathrm{m}$.

dure. Photoreceptor synapses were strongly enriched in the OPLfraction (Fig. 5c) in comparison with the crude homogenate (Fig. $5 a$ ) and the CSM-fraction (Fig. 5b).

This enrichment in synapses of the OPL-fraction was also confirmed by immunoblotting with antibodies against two synapse markers and one glial cell marker (Fig. 6). Antibodies to synaptophysin (Wiedenmann and Franke, 1985) and antibodies to SP14 that have been used as synapse markers in various regions of the CNS (Honer et al., 1993; Simpson et al., 1994) became strongly enriched in the OPL-fraction, thus indicating further the enrichment of synapses in the OPL-fraction (Fig. 6). The GFAP that is not a component of photoreceptor synapses but is expressed in astrocytes and Müller cells (Erickson et al., 1993) was not detected in the OPL-fraction (Fig. 6C).

\section{Purification of synaptic ribbons from the OPL-fraction}

The OPL-fraction was subjected to Triton X-100 extraction and sucrose density fractionation. The resulting subfractions were screened for the presence of synaptic ribbons. Figure $7 a$ is a cryostat section of the fraction between sucrose steps $50 \%$ - and

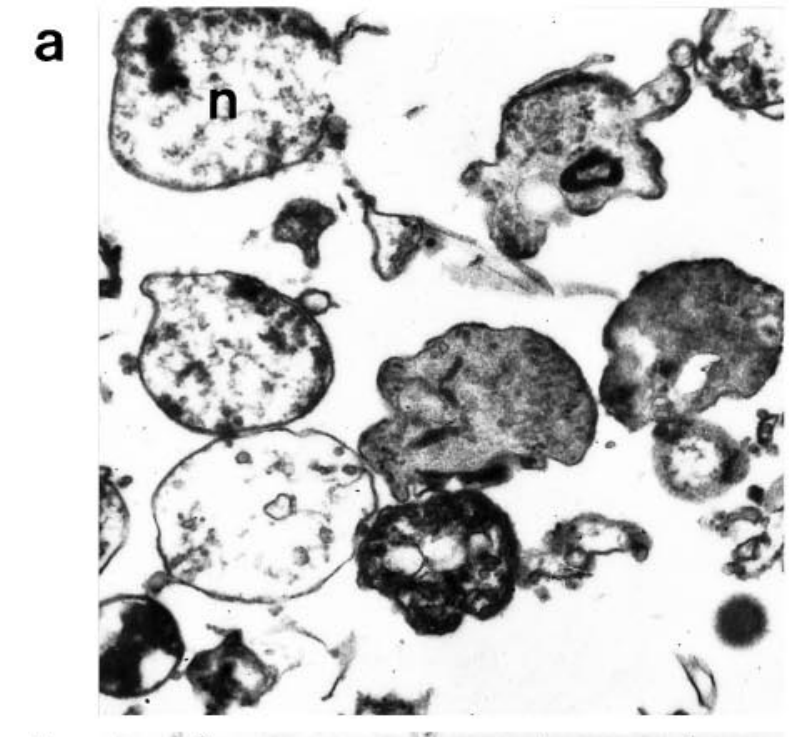

b
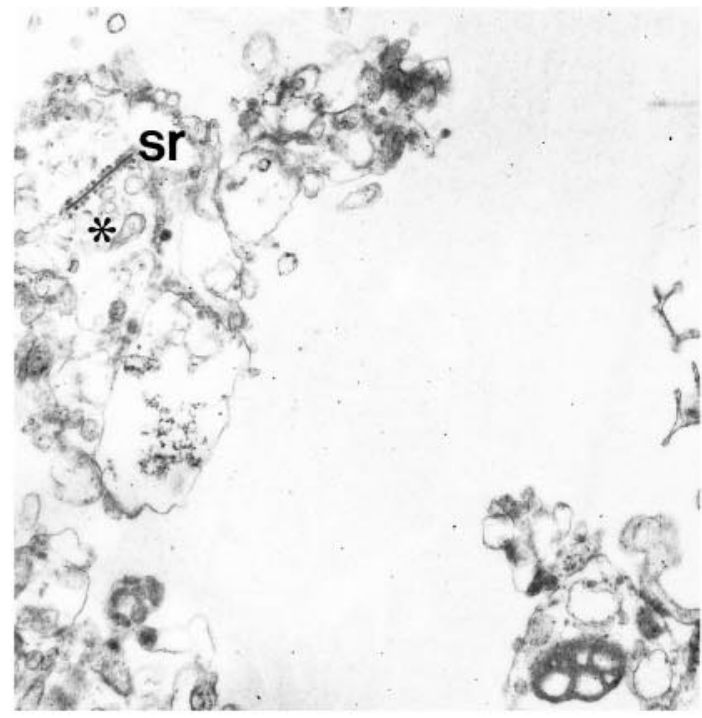

C

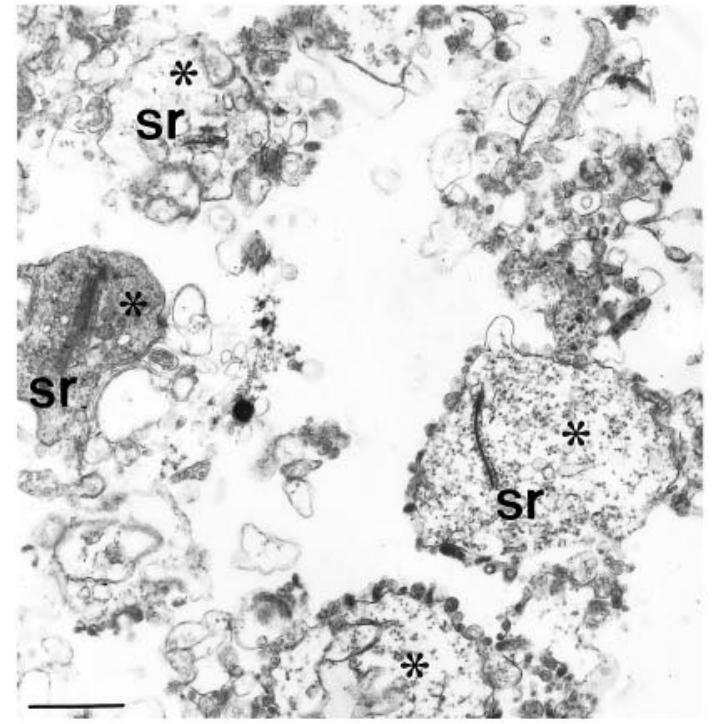

Figure 5. $a-c$, Ultrastructural analysis of the crude homogenate $(a)$, the CSM-fraction $(b)$, and the OPL-fraction $(c)$. In the OPL-fraction, note the presence of many typically shaped photoreceptor synapses (asterisks) containing synaptic ribbons. $s r$, Synaptic ribbon; $n$, nucleus. Scale bar, $625 \mathrm{~nm}$. 


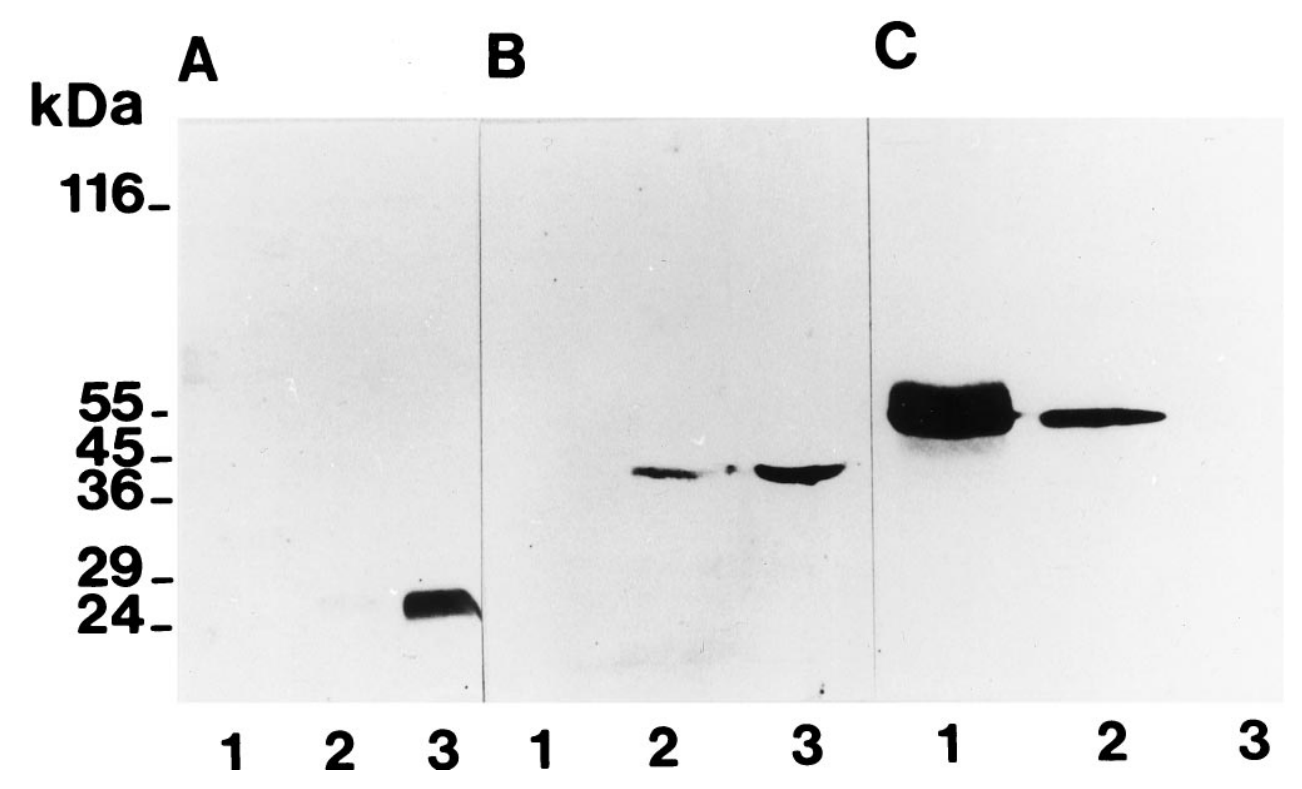

Figure 6. $a-c$, Immunoblotting of different membrane fractions using antibodies specific for two synaptic $(A, \mathrm{SP} 14 ; B$, synaptophysin) and one nonsynaptic marker proteins $(C$, glial fibrillary acidic protein) Lane 1 is loaded with the starting material (crude homogenate), lane 2 with the CSMfraction, and lane 3 with the purified photoreceptor synapse fraction (OPL-fraction) Equal amounts of proteins were applied to these three lanes $(2 \mu \mathrm{g}$ on each lane for the synaptophysin immunoblot, $25 \mu \mathrm{g}$ on each lane for the SP14 immunoblot, and $50 \mu \mathrm{g}$ on each lane for the GFAP immunoblot).

$70 \%$-immunolabeled with the ribbon antiserum. Very obvious is the high density of synaptic ribbons present in the SR-fraction that exceeded considerably the density of synaptic ribbons observed in all other fractions, including the OPL-fraction (Fig. 4). The SRfraction accounted for $\sim 25 \%$ of total Triton-resistant protein. Conventional transmission electron microscopy of this fraction (Fig. $7 b, c$ ) demonstrated various cross-sections of synaptic ribbons and dense material surrounding them. Preembedding immunoelectron microscopy of the SR-fraction labeled with the ribbon antiserum showed that not only the bar-shaped structures were labeled with the ribbon antiserum but also the surrounding dense material, indicating that this material originates from disassembled ribbons (Fig. 7d). Therefore, we conclude that the SRfraction is highly enriched in photoreceptor synaptic ribbons and their disassembly products. In addition to the ribbon-like structures, the SR-fraction contained some membranous structures that were not labeled by the ribbon antiserum. These might represent Triton X-100-insoluble components of the presynaptic terminal and/or postsynaptic dendrites that are associated with synaptic ribbons.

SDS-PAGE of the SR-fraction (Fig. 8, lane 3) revealed several major polypeptide bands. The most prominent bands were found at $\sim 240,60,55,43$, and $30 \mathrm{kDa}$. Three of these major bands of the SR-fraction reacted strongly with antibodies against brain spectrin $(240 \mathrm{kDa})$, tubulin $(55 \mathrm{kDa})$, and actin (43 kDa) (data not shown).

All attempts to identify the component that is recognized by the ribbon antiserum failed. The antibody did not react in Western blots and could also not be used for immunoprecipitation.

\section{DISCUSSION}

The purpose of the present study was the development of a procedure for the isolation and purification of synaptic ribbons from photoreceptor synapses. Because the synaptic ribbon is considered to be important for vesicle clustering at the active zones of photoreceptor synapses (for review, see Burns and Augustine, 1995), this question is of considerable biological relevance for understanding the molecular anatomy of the active zones of photoreceptor synapses. To achieve this aim we used a two-step procedure: (1) purification of photoreceptor synapses and (2) isolation of synaptic ribbons from this purified photoreceptor synapse fraction. As judged by various techniques (immunofluorescence microscopy, electron microscopy, immunoelectron microscopy), we obtained a fraction that was highly enriched in synaptic ribbons and their disassembly products.

The purification of isolated photoreceptor synapses in the OPL-fraction was controlled by different procedures. (1) In the electron microscope, photoreceptor synapses could be recognized easily because of their typical size and typical structural features (large synaptic ribbons, invagination of the presynaptic plasma membrane). Photoreceptor synapses were clearly enriched in the OPL-fraction, as judged by comparison of lowpower micrographs of the OPL-fraction with the crude homogenate and the CSM-fraction. (2) With use of immunofluorescence microscopy with the ribbon antiserum, the OPL-fraction was strongly enriched in large horseshoeshaped synaptic ribbons that are typical for photoreceptor synapses and not found elsewhere in the retina. Synaptic ribbons in synapses of the IPL are much smaller in size (for reviews, see Hogan et al., 1971; Rodieck, 1973; Dowling, 1987) and appeared as small, dot-like immunolabeled structures (Fig. $3 b$ ). Synapses from the IPL thus could be clearly distinguished from the much larger synapses of the OPL because of the larger size and shape of the synaptic ribbons in immunofluorescence microscopy. Furthermore, synapses from the IPL of the retina (identified by staining with the ribbon antiserum as dot-shaped but not horseshoe-shaped structures) fractionated at a different sucrose density [density of $\sim 36 \%$ sucrose $(w / v)$ ] than do the large photoreceptor synaptic complexes (not shown).

The OPL-fraction was also enriched in general synapse markers (synaptophysin, SP14) and showed virtually no contamination with fragments of Müller cells, as indicated by Western blotting using antibodies to GFAP.

(2) From this OPL-fraction we prepared a Triton X-100resistant subfraction, the SR-fraction, in which synaptic ribbons were the main component. Transmission electron microscopy showed many cross-sections of synaptic ribbons and also large amounts of electron-dense material surrounding them. This 


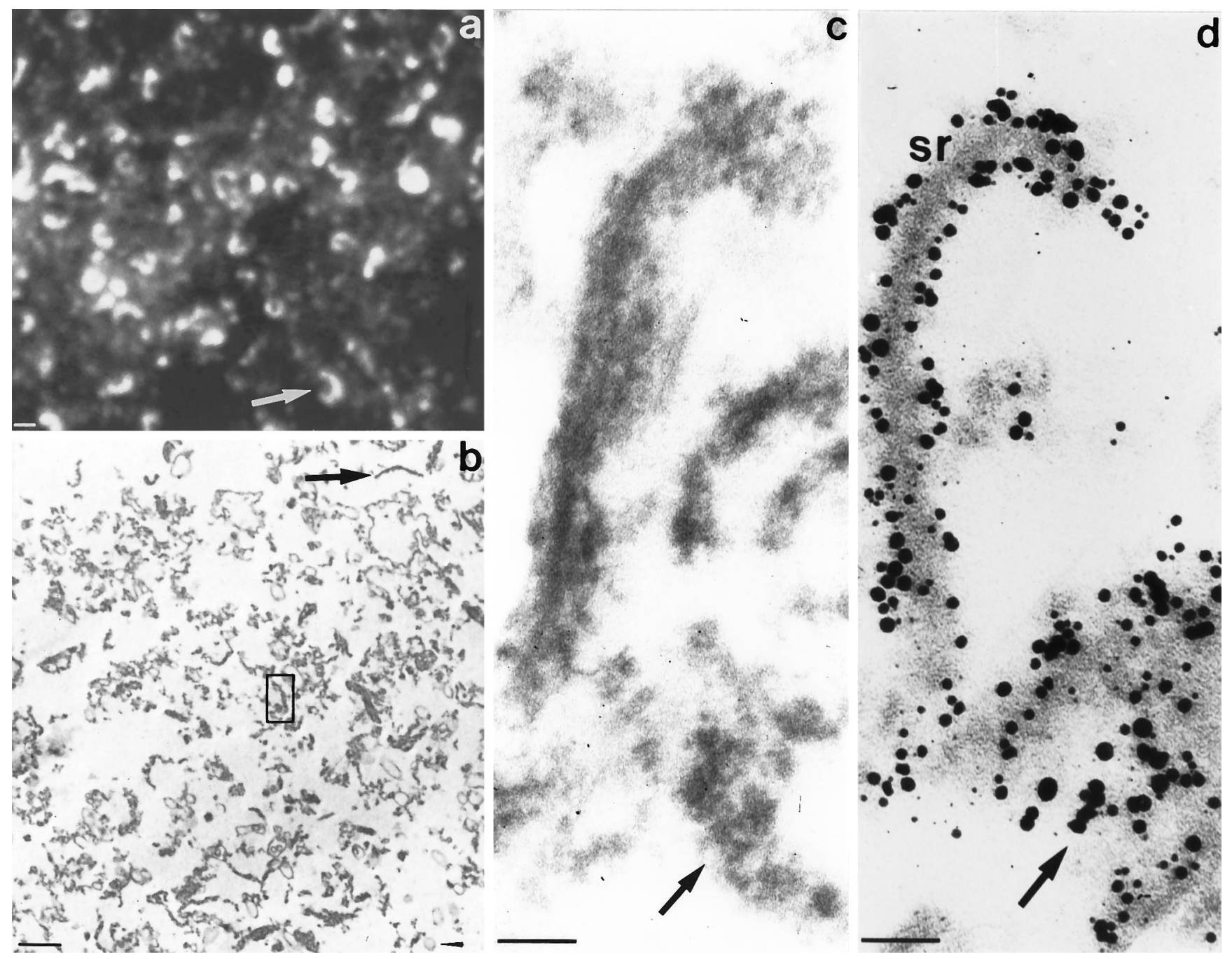

Figure 7. $a-d$, Characterization of the SR-fraction. $a$ shows a cryostat section of the SR-fraction labeled with the ribbon antiserum and processed for immunofluorescence and confocal laser scanning microscopy. Note the high density of horseshoe-shaped synaptic ribbons present in this fraction. $b$ represents a conventional transmission electron micrograph of the SR-fraction. Note the presence of many bar-like ribbon profiles (arrow) surrounded by electron-dense material. Only a few contaminating membranous structures were present in this fraction (arrowhead). $c$ is a higher, enlarged transmission electron micrograph similar to the one labeled in $b(b o x$ in $b)$. Note the bar-shaped ribbon profile surrounded by electron-dense material. The isolated synaptic ribbons appear more diffuse than ribbons in situ. In $d$, the SR-fraction was immunolabeled with the ribbon antiserum to analyze which components of the SR-fraction represent ribbon material. Both ribbons as well as the surrounding electron-dense material are immunolabeled with the ribbon antiserum, indicating that the dense material represents a disassembly product of synaptic ribbons. Scale bars: $a, b, 1 \mu \mathrm{m} ; c, d, 100 \mathrm{~nm}$.

dense material seemed to originate from synaptic ribbons: immunoelectron microscopy revealed that both the bar-shaped ribbon profiles and the dense material were immunoreactive with the ribbon antiserum. Therefore, it is likely that the dense material originated from disassembled synaptic ribbons. In situ, it has been known that the ribbon is able to disassemble into smaller subunits (Wagner, 1973; Schmitz et al., 1989; Schmitz and Drenckhahn, 1993). SDS-PAGE of the SR-fraction revealed several major polypeptide bands. These major polypeptide bands of the ribbon fraction migrated at $\sim 240,60,55,43$, and $30 \mathrm{kDa}$.

Because the SR-fraction has been shown by the abovementioned, independent methods to be strongly enriched in synaptic ribbons, it seems reasonable to assume that the yet unknown components of synaptic ribbons are among the polypeptide bands contained in the SR-fraction. If synaptic ribbons turn out to be polymeric structures that assemble from a few bulk proteins, as do microtubules that consist of $\alpha, \beta$-tubulin dimers and a few microtubule-associated proteins, one could expect that the major polypeptide bands of the SR-fraction might represent the building blocks of synaptic ribbons. If instead synaptic ribbons are heteropolymers consisting of several proteins, the minor polypeptide bands of the SR-fraction also have to be taken into account as constituents of the ribbons.

In conclusion, in the present study photoreceptor synaptic ribbons have been isolated for the first time. Because a procedure for the isolation of synaptic ribbons has been established, a methodological basis now is available for the molecular and functional characterization of this synaptic organelle. This might be of importance not only for understanding the molecular anatomy of the active zone complex in photoreceptors, but it might also help to identify components of presynaptic densities found in other synapses of the CNS and peripheral nervous system. 


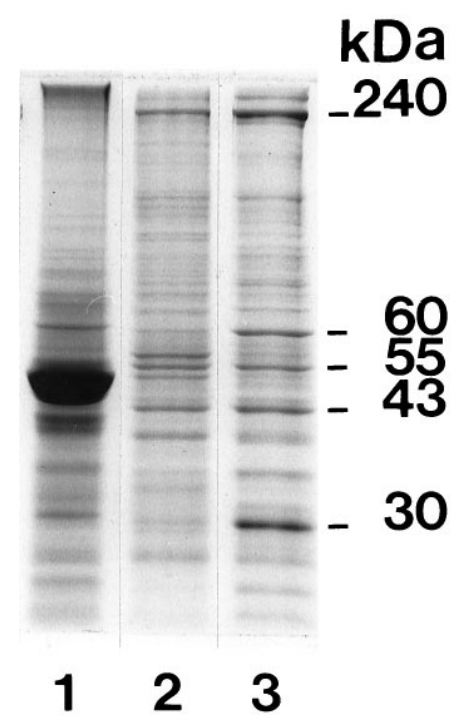

Figure 8. SDS-PAGE (10\% acrylamide) of various retinal fractions loaded with equal amounts of total proteins. Lane 1, crude retinal homogenate (starting material); lane 2, OPL-fraction; lane 3, SR-fraction. The SR-fraction contains five major polypeptide bands that migrated at 240, $60,55,43$, and $30 \mathrm{kDa}$. Twenty micrograms of protein were applied on each lane.

\section{REFERENCES}

Ahnelt P, Keri C, Kolb H (1990) Identification of pedicles of putative blue-sensitive cones in the human retina. J Comp Neurol 293:39-53.

Burns ME, Augustine GJ (1995) Synaptic structure and function. Cell 83:187-194.

Danscher G (1981) Localization of gold in biological tissues. Histochemistry $71: 81-88$.

Dieckmann-Schuppert A, Schnittler H-J (1997) A simple assay for quantitation of protein in tissue sections, cell cultures, cell homogenates and proteins immobilized on solid surfaces. Cell Tissue Res, in press.

Dowling JE (1987) Retinal synapses. In: The retina: an approachable part of the brain (Dowling JE, ed). Cambridge, MA: Belknap.

Drenckhahn D, Schlüter K, Allen DP, Bennett V (1985) Colocalization of band 3 with ankyrin and spectrin at the basal membrane of intercalated cells in the rat kidney. Science 230:1287-1289.

Erickson PA, Lewis GP, Fisher SK (1993) Postembedding immunocytochemical techniques for light and electron microscopy. Methods Cell Biol 37:283-308.

Giloh H, Sedat JW (1982) Fluorescence microscopy: reduced photobleaching of rhodamine and fluorescein protein conjugates by n-propyl gallate. Science 217:1252-1255.

Heinzel W, Vogt A, Kallee E, Faller W (1965) A new method for the quantitative determination of antibody and antigen protein, with a sensitivity of five micrograms. J Lab Clin Med 66:334-340.
Hogan MJ, Alvarado JA, Weddell JE (1971) Histology of the human eye. An atlas and textbook. Philadelphia: W. B. Saunders.

Honer WG, Lu L, Davies P (1993) Human synaptic proteins with a heterogeneous distribution in cerebellum and visual cortex. Brain Res 609:9-20.

Laemmli UK (1970) Cleavage of structural proteins during the assembly of the head of the bacteriophage T4. Nature 227:680-685.

McCartney MD, Dickson DH (1985) Photoreceptor synaptic ribbons: three-dimensional shape, orientation and diurnal (non) variation. Exp Eye Res 41:313-321.

McLaughlin BJ, Boykins L (1977) Ultrastructure of E-PTA stained synaptic ribbon in the chick retina. J Neurobiol 8:91-96.

Olmstedt JB (1981) Affinity purification of antibodies from diazotized paper blots of heterogeneous protein samples. J Biol Chem 256:11955-11957.

Rao-Mirotznik R, Harkins AB, Buchsbaum G, Sterling P (1995) Mammalian rod terminal: architecture of a binary synapse. Neuron 14:561-569.

Raviola E (1976) Intercellular junctions in the outer plexiform layer of the retina. Invest Ophthalmol 15:881-895.

Rodieck RW (1973) The vertebrate retina. San Francisco: W. H. Freeman.

Rothman JE (1994) Mechanisms of intracellular protein transport. Nature 372:55-63.

Schaeffer SF, Raviola E (1978) Membrane recycling in the cone cell endings in the turtle retina. J Cell Biol 79:802-825.

Schaeffer SF, Raviola E, Heuser JE (1982) Membrane specializations in the outer plexiform layer of the turtle retina. J Comp Neurol 204:253-270.

Schmitz F, Drenckhahn D (1993) Intermediate stages in the disassembly of synaptic ribbons in cone photoreceptors of the crucian carp, Carassius carassius. Cell Tissue Res 272:487-490.

Schmitz F, Kirsch M, Wagner H-J (1989) Calcium-modulated synaptic ribbon dynamics in cone photoreceptors: a pharmacological and electron spectroscopic study. Eur J Cell Biol 49:207-212.

Schmitz F, Anderson LVB, Drenckhahn D (1995) Characterization of structural proteins of the photoreceptor synapse. J Cell Biochem [Suppl] 19B:B110 (Abstr).

Simpson IA, Chundu KR, Davies-Hill T, Honer W-G, Davies P (1994) Decreased concentrations of GLUT1 and GLUT3 glucose transporters in the brains of patients with Alzheimer's disease. Ann Neurol 35:546-551.

Sjöstrand FS (1958) Ultrastructure of retinal rod synapses of the guinea pig eye as revealed by three-dimensional reconstructions from serial sections. J Ultrastruct Res 2:122-170.

Towbin H, Staehelin T, Gordon J (1979) Electrophoretic transfer of proteins from polyacrylamide gels to nitrocellulose sheets: procedure and some applications. Proc Natl Acad Sci USA 76:4350-4354.

Wagner H-J (1973) Darkness-induced reduction of the number of synaptic ribbons in fish retinae. Nature (New Biol) 246:53-55.

Wiedenmann B, Franke WW (1985) Identification and localization of synaptophysin, an integral membrane glycoprotein of $\mathrm{Mr} 38,000$, characteristic of presynaptic vesicles. Cell 41:1017-1021. 